\title{
Laparoscopic liver resection: Global diffusion and learning curve
}

\author{
Epameinondas Dogeas ${ }^{1} M D$, Samer Tohme ${ }^{1} M D$, David A Geller ${ }^{1} M D$
}

Laparoscopic liver resection (LLR) is being utilised with increasing frequency worldwide, as initial concerns about the safety and feasibility of LLR, such as the risk of uncontrolled major haemorrhage and potential compromise of oncologic outcomes, were not supported by the data. On the contrary, LLR was found to be associated with several significant perioperative benefits compared to open liver resection, including less blood loss, less narcotic requirement, fewer complications, and reduced hospital stay. ${ }^{1,2}$ Knowledge of precision liver anatomy remains paramount to performing anatomically correct LLR both for hepatocellular carcinoma and colorectal cancer liver metastasis. This will achieve adequate oncologic outcomes, as outlined in the recent international consensus conference on precision anatomy for minimally invasive hepatopancreatobiliary surgery in Tokyo, Japan. ${ }^{3-6}$ The increased utilisation of LLR is reflected in 2 worldwide literature reviews published only 7 years apart; the earlier one by Nguyen et al. in 2009 captured 2,804 patients, while a subsequent study in 2016 by Ciria et al. contained over triple the number of patients $(n=9,527) .{ }^{1,2}$ Robotic liver resection (RLR) has also emerged as a safe alternative to open liver resection with short-term clinical benefits. However, when comparing LLR to RLR, perioperative and longterm outcomes appear to be equivalent, while the cost of RLR is higher compared to laparoscopy. ${ }^{7,8}$

The global dissemination of LLR was highlighted by Hibi et al. in 2014 in a survey that revealed LLR was routinely performed in more than 40 countries across Asia, Europe and the Americas. ${ }^{9}$ Their survey also revealed that the indications of LLR were expanding, as half of the participating centres were routinely performing LLR for either major hepatectomy or for resection of "difficult" posterosuperior liver segments. ${ }^{10}$ The global diffusion of difficult LLR has continued to expand following the 2nd International Consensus Conference on Laparoscopic Liver Resection, with Ibuki et al. reporting on 4,478 difficult LLR patients from 58 centres in 19 countries between 2014 and 2018. ${ }^{11}$ Notably, as minimally invasive donor hepatectomy is proliferating, a set of expert consensus guidelines was established to guide its safe implementation. ${ }^{12}$ Today, worldwide interest for LLR remains strong as evidenced by the attendance of the recent International Laparoscopic Liver Society (ILLS) 2021 3rd World Congress live virtual meeting that convened in June 2021. The meeting had 1,357 registrants from 86 countries and territories and territories (Fig. 1). The number of registrants per continent is shown, with Asia having the most at 422 registrants, followed by Europe with 329, North America with 291, and South America with 272. The most number of registrants came from the US, Brazil, Mexico and Japan. One of the challenges in diffusing technology-dependent procedures such as LLR is the ability to implement them in low-resource countries due to lack of equipment availability and cost. Noteworthy is the participation of liver surgeons at the ILLS 2021 meeting from many low-resource countries including Bangladesh, Burundi, Costa Rica, Croatia, Estonia, Guatemala, Iran, Jamaica, Jordan, Lebanon, Lithuania, Maldives, Nigeria, Qatar, Saudi Arabia, Slovenia, Tunisia and Yemen. This is highly indicative of global diffusion of laparoscopic liver surgery interest (and hopefully utilisation) around the world.

The growth in number and complexity of LLR and its adoption by surgeons without prior experience in minimally invasive liver surgery prompted efforts to delineate the learning curve of LLR, defined as improvement in performance over time. Performance metrics that have been used in the LLR learning curve literature included operating room (OR) time, intraoperative estimated blood loss, conversion to open hepatectomy and postoperative morbidity. However, not all studies report on all the above parameters and therefore one must exercise caution when comparing between different learning curve series. Furthermore, the learning curve will vary based on case difficulty and the surgeon's prior experience with open hepatobiliary surgery and minimally invasive surgery. ${ }^{13,14}$ Vigano et al. looked into the need for conversion to an open approach and discovered that the learning curve of laparoscopic minor hepatectomy for a self-taught surgeon is 60 cases. ${ }^{15}$ Regarding laparoscopic major hepatectomy, Nomi et al. focused

${ }^{1}$ Division of Hepatobiliary and Pancreatic Surgery, Department of Surgery, University of Pittsburgh Medical Center, USA

Correspondence: Dr David A Geller, UPMC Montefiore, 7 South, 3459 Fifth Ave, Pittsburgh, PA 15213-2582, USA.

Email: gellerda@upmc.edu 


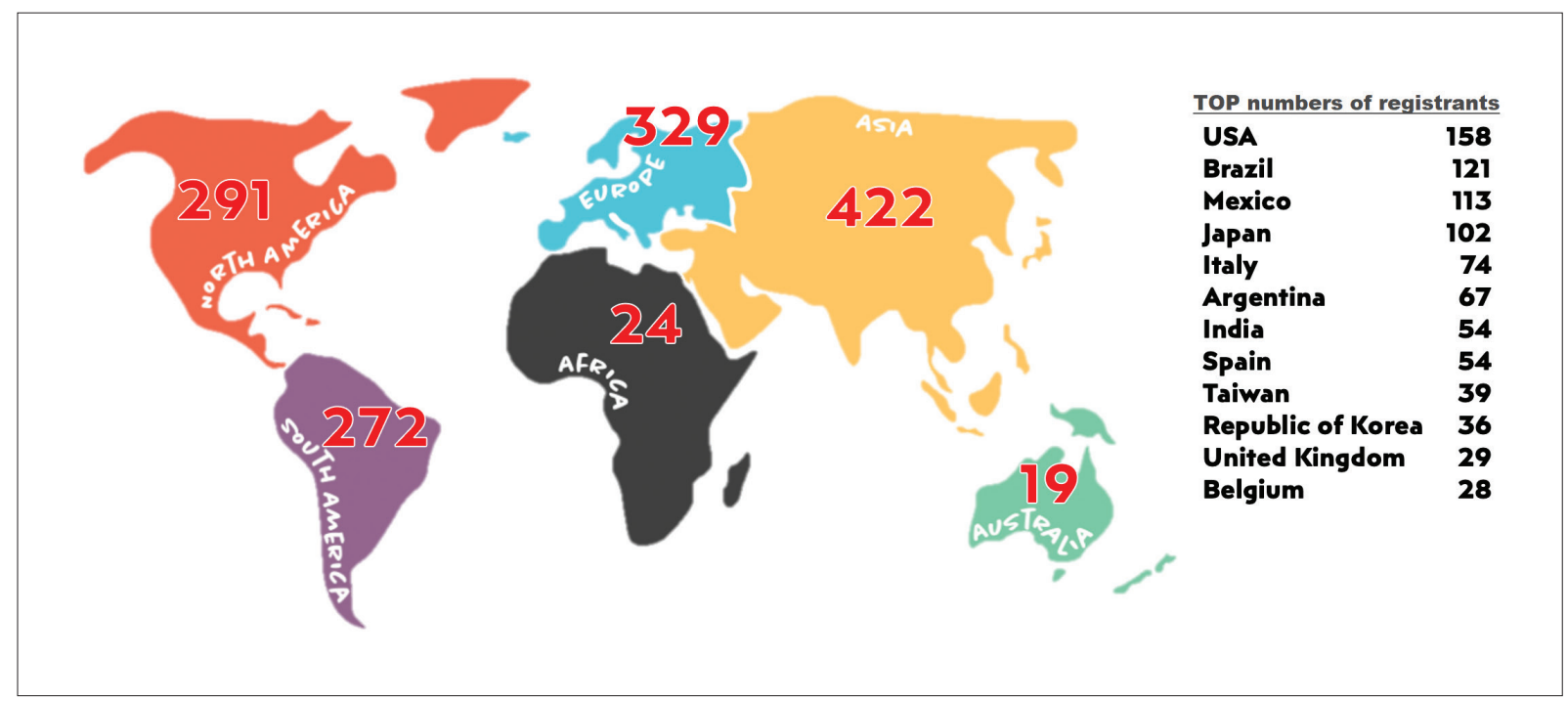

Fig. 1. Global map of registrants for the International Laparoscopic Liver Society 2021 3rd World Congress live virtual meeting that convened in June 2021. The meeting had 1,357 registrants from 86 countries and territories.

on OR time as a metric in 173 laparoscopic major hepatectomies and found that their learning curve spanned $45-75$ cases. ${ }^{16}$ Their findings were corroborated by van der Poel et al. who looked into their own 159 laparoscopic major hepatectomies, and using the need for conversion as a metric, defined their learning curve as 55 cases. $^{17}$

In one of the largest LLR series published to date, Swaid et al. reported on the University of Pittsburgh Medical Center experience with 1,062 LLR patients spanning almost 2 decades (2001-2017). ${ }^{18}$ The authors examined performance over time and divided the study cohort into 3 periods: 2001-2007 $(n=203)$, 2008-2012 $(\mathrm{n}=426)$ and 2013-2017 $(\mathrm{n}=433)$. They found that over the study period, a greater percentage of overall hepatectomies were performed laparoscopically despite operating on more patients with background liver cirrhosis. Furthermore, a greater percentage of LLR were accomplished in a pure laparoscopic fashion, while OR time, need for transfusion and postoperative complications decreased. Analysing the literature, cumulative sum analysis factoring OR time and the need for conversion revealed that the learning curve exhibited 2 phases; 45 cases were required to break through the initial learning curve, but a total of 60-70 cases were required to further progress into the standardisation "mastery" phase that includes major hepatectomy and difficult segments. Therefore, the authors recommend that surgeons, who are still in their initial learning curve, start with laparoscopic minor hepatectomy, including left lateral sectionectomy, and after 45 cases they can progress to laparoscopic major hepatectomy or partial hepatectomy of the difficult posterosuperior segments. More complex resections, such as laparoscopic live-donor hemi-hepatectomy, should be undertaken only when the "mastery" phase is reached.

In this issue of the Annals, Goh et al. report on a sizable single surgeon series of 310 consecutive patients who underwent LLR from 2011 to $2021 .{ }^{19}$ Of note, although the surgeon had no prior experience in LLR, he was experienced in open hepatobiliary surgery and complex minimally invasive abdominal surgery. The study data encompass all relevant clinicopathologic factors, including the Iwate and Institut Mutualiste Montsouris (IMM) difficulty score grading of the resections, and all pertinent perioperative metrics are reported, such as OR time, blood loss, need for transfusion, conversion to open approach and morbidity. The authors report excellent outcomes, with a low rate of margin positive resection (3.1\%) and only 2 in-hospital mortalities in the entire cohort. As the existing literature points to about a 60-case requirement to navigate the LLR learning curve, the authors further subdivided their cohort in 5 chronologically consecutive groups of 60 patients each. They discovered that over time, LLR was performed on higher risk patients (older age, higher American Society of Anesthesiologists score, and increased frequency of portal hypertension) requiring technically more demanding resections (larger tumour size). Despite this, they noted improved outcomes over the study 
period: decrease in the open conversion rate, blood loss and blood transfusion rate. The importance of the initial LLR learning curve was again demonstrated in the analysis of factors associated with conversion to an open approach, where only surgeon experience with $<60$ cases and IMM difficulty score of III were independent predictors of open conversion on multivariate analysis.

In summary, LLR is being increasingly utilised across the world. The initial learning curve for surgeons without previous experience in LLR is steep and requires 45-70 cases. Increased surgeon experience is associated with improvement in perioperative outcomes despite operating on higher-risk patients and performing more complex resections. It is likely that the learning curve of the "second generation" aparoscopic liver surgeons who are trained by surgeons already experienced in LLR will be shorter, but that remains to be studied.

\section{REFERENCES}

1. Nguyen KT, Gamblin TC, Geller DA. World review of laparoscopic liver resection - 2,804 patients. Ann Surg 2009;250:831-41.

2. Ciria R, Cherqui D, Geller DA, et al. Comparative Short-term Benefits of Laparoscopic Liver Resection. Ann Surg 2016;263:761-77.

3. Ciria R, Berardi G, Nishino H, et al. A snapshot of the 2020 conception of anatomic liver resections and their applicability on minimally invasive liver surgery. A preparatory survey for the Expert Consensus Meeting on Precision Anatomy for Minimally Invasive HBP Surgery. J Hepatobiliary Pancreat Sci 2021. doi:10.1002/jhbp.959.

4. Morimoto M, Tomassini F, Berardi G, et al. Glissonean approach for hepatic inflow control in minimally invasive anatomic liver resection: A systematic review. J Hepatobiliary Pancreat Sci 2021. doi:10.1002/jhbp. 908 .

5. Monden K, Alconchel F, Berardi G, et al. Landmarks and techniques to perform minimally invasive liver surgery: A systematic review with a focus on hepatic outflow. J Hepatobiliary Pancreat Sci 2021. doi: $10.1002 / \mathrm{jhbp} .898$

6. Wakabayashi T, Cacciaguerra AB, Ciria R, et al. Landmarks to identify segmental borders of the liver: A review prepared for PAM-HBP expert consensus meeting 2021. J Hepatobiliary Pancreat Sci 2021. doi:10.1002/jhbp.899.
7. Ziogas IA, Evangeliou AP, Mylonas KS, et al. Economic analysis of open versus laparoscopic versus robotic hepatectomy: a systematic review and meta-analysis. European J Heal Econ 2021;22:585-604.

8. Ziogas IA, Giannis D, Esagian SM, et al. Laparoscopic versus robotic major hepatectomy: a systematic review and meta-analysis. Surg Endosc 2021;35:524-35

9. Hibi T, Cherqui D, Geller DA, et al. International Survey on Technical Aspects of Laparoscopic Liver resection: a web-based study on the global diffusion of laparoscopic liver surgery prior to the 2nd International Consensus Conference on Laparoscopic Liver Resection in Iwate, Japan. J Hepatobiliary Pancreat Sci 2014;21:737-44

10. Hibi T, Cherqui D, Geller DA, et al. Expanding indications and regional diversity in laparoscopic liver resection unveiled by the International Survey on Technical Aspects of Laparoscopic Liver Resection (INSTALL) study. Surg Endosc 2016;30:2975-83.

11. Ibuki S, Hibi T, Tanabe M, et al. Short-term Outcomes of "Difficult" Laparoscopic Liver Resection at Specialized Centers: Report From INSTALL (International Survey on Technical Aspects of Laparoscopic Liver Resection)-2 on 4478 Patients. Ann Surg 2020. doi:10.1097/SLA.0000000000004434

12. Cherqui D, Ciria R, Kwon CHD, et al. Expert Consensus Guidelines on Minimally Invasive Donor Hepatectomy for Living Donor Liver Transplantation From Innovation to Implementation: A Joint Initiative From the International Laparoscopic Liver Society (ILLS) and the Asian-Pacific Hepato-Pancreato-Biliary Association (A-PHPBA). Ann Surg 2021;273:96-108.

13. Cheek SM, Geller DA. The Learning Curve in Laparoscopic Major Hepatectomy: What Is the Magic Number? JAMA Surg 2016;151:929.

14. Brown KM, Geller DA. What is the Learning Curve for Laparoscopic Major Hepatectomy? J Gastrointest Surg 2016; 20:1065-71.

15. Vigano L, Laurent A, Tayar C, et al. The Learning Curve in Laparoscopic Liver Resection. Ann Surg 2009;250:772-82.

16. Nomi T, Fuks D, Kawaguchi Y, et al. Learning curve for laparoscopic major hepatectomy. Brit J Surg 2015;102:796-804.

17. MJ van der Poel, Besselink MG, Cipriani F, et al. Outcome and Learning Curve in 159 Consecutive Patients Undergoing Total Laparoscopic Hemihepatectomy. JAMA Surg 2016;151:923.

18. Swaid F, Sucandy I, Tohme S, et al. Changes in Performance of More Than 1000 Minimally Invasive Liver Resections. JAMA Surg 2020;155:986-8

19. Goh BKP, Wang Z, Koh YX, et al. Evolution and trends in the adoption of laparoscopic liver resection in Singapore: Analysis of 300 cases. Ann Acad Med Singap 2021;50:742-50. 